\title{
Effects of social distancing on psychological state and physical activity routines during the COVID-19 pandemic
}

\author{
Donatella Di Corrado ${ }^{1,5} \cdot$ Paola Magnano ${ }^{1} \cdot$ Benedetta Muzii ${ }^{2} \cdot$ Marinella Coco ${ }^{3} \cdot$ Maria Guarnera $^{1} \cdot$ \\ Salvatore De Lucia ${ }^{4} \cdot$ Nelson Mauro Maldonato ${ }^{4}$
}

Received: 15 July 2020 / Accepted: 29 August 2020 / Published online: 25 September 2020

(c) Springer-Verlag Italia S.r.l., part of Springer Nature 2020

\begin{abstract}
Background The novel coronavirus disease (COVID-19) has affected the entire world. Since the reporting of the first cases, Italy has quickly become the country hit second firmest in the world by the coronavirus. Governments' immediate protective restrictions modified the habit of the individuals and included full lockdowns of cities, travel, restricted social congregations, and suspended schools.

Objectives The aim of this study was to survey the general community in Italy to better recognize their levels of psychological impact, emotional responses and maintaining their daily exercise or physical activity routines during the initial stage of the COVID-19 outbreak.

Methods 670 adults were invited to complete an online survey collecting information on demographic data, physical and emotional symptoms in the past 14 days, contact history with COVID-19, and keeping regular physical activity. Data analysis was conducted through mixed qualitative and quantitative methods.

Results During the initial phase of the COVID-19 outbreak in Italy, more than half of the respondents reported a significant psychological and physical impact.

Conclusion The COVID-19 pandemic is bringing significant challenges to people, families, and countries. For further studies, these findings can be used to advance psychological interventions to reduce the consequences of the COVID-19 pandemic.
\end{abstract}

Keywords Coronavirus · Psychological impact $\cdot$ Physical activity $\cdot$ Lockdown

\section{Introduction}

In December 2019, cases of life-threatening pneumonia were testified in Wuhan, China. A novel coronavirus (COVID-19) was recognized as the cause of infection, with the ability

Donatella Di Corrado

donatella.dicorrado@unikore.it

1 Faculty of Human and Social Sciences, Kore University of Enna, 94100 Enna, Italy

2 Department of Humanistic Studies, University of Naples Federico II, Naples, Italy

3 Department of Biomedical and Biotechnological Sciences, University of Catania, Catania, Italy

4 Department of Neuroscience and Reproductive and Odontostomatological Sciences, University of Naples Federico II, Naples, Italy

5 Department of Sport Sciences, Kore University of Enna, Cittadella Universitaria, 94100 Enna, Italy to penetrate the Central Nervous System via the olfactory neural pathway. The number of reported cases has rapidly increased, affecting the entire world. On 30 January 2020, the World Health Organization (WHO) confirmed this disease a 'public health emergency of international concern.'

In Italy, since the first case of COVID-19 (February 20th, 2020), a fast range of the contagions was testified in the first weeks of March. Italy has quickly become the country hit second firmest in the world by the coronavirus. Reducing physical interaction, commonly known as 'social distancing' has appeared as the first line of defense fight against this pandemic [1].

Since coronavirus infected individuals can transmit it for up to 5 days before their symptoms manifest, limiting social interactions between even apparently healthy people stands to slow the circulation of the virus. For this reason, governments' immediate protective restrictions included full lockdowns of cities, travel, restricted social congregations, including sports events, concerts, restaurants, and the 
closing of schools and universities [2]. These procedures changed radically the routine of the individuals, imposing a large burden on families, whereby parents and children stayed at home for the entire day. The pressures of longterm co-residence in close internment are not to be reduced, particularly when parents must associate the demands of working from home with those of caring for children. For the first time since the end of the Second World War, the Italian population was faced with a limitation in freedom of movement, having catastrophic effects on the economy, as well as social effects on the people. Obviously, a period of quarantine was the best decision to stop the rapid spread infections [3]. However, this may have had collateral effects on other dimensions of the individuals' psychological and physical health. Quarantine means separation from loved ones, the deprivation of freedom, restriction of movement, uncertainty over disease status, loneliness, and boredom can be an unpleasant experience for the population.

Those who live alone are most affected, deprived as they will be of the numerous benefits that come from physical co-presence with others. The probable benefits of mandatory mass quarantine as a public health measure need to be assessed closely against the possible psychological costs. Earlier studies on several epidemics have highlighted psychological consequences on individuals because they include dissimilar levels of social life. The sudden circulation of an unknown virus and the measures adopted to reduce its spread have had a strong effect on the quality of life of the people $[4,5]$.

A recent review [6] examined the psychological impact of quarantine, reporting stress symptoms, confusion, anger, infection fears, frustration, and boredom. Other researchers concluded that quarantine and isolation could lead to anxiety and depression [7, 8]. Moreover, the prolonged home stay in many cases may include a reduction of the level of physical activity (hence lower energy expenditure) to maintain an adequate health status [9]. It is likely that the change of lifestyles may lead to increased sedentary behaviors, such as spending excessive amounts of time sitting (working from home), or lying down for screening activities (playing games, watching television, using mobile devices), with a subsequent impact on physical well-being. The positive benefits of regular physical activity on many health outcomes are well established [10,11]. Moreover, maintaining a regular exercise and preserving lifestyle during self-isolation is very important for the prevention of future chronic health conditions due to a sedentary habits. However, a strong motivation for continuing physical activity in the home is needed.

Currently, low information is reported on the general psychological impact of the COVID-19 epidemic and the maintaining daily exercise or physical activity routines during the initial stage of the outbreak. Our goal was to offer a looking of the Italian condition in the first weeks of the restrictive procedures defined by the severe diffusion of the COVID-19. The aim of this study was to survey the general community in Italy to better recognize their levels of psychological impact, emotional responses and maintaining their daily exercise or physical activity routines during the initial stage of the COVID-19 outbreak. In particular, this study aims to detect whether change or maintenance of physical activity habits shows a relationship with psychological and physical aspects.

\section{Methods}

\section{Sample}

This study was carried out in accordance with the recommendations of the Ethical Code of the University of Palermo and of the Code of Ethics approved by the General Assembly of the Italian Association of Psychology held on March 27, 2015. All participants were informed about the trials of the study and the anonymity of their answers before providing their written consent to participate, in accordance with the Declaration of Helsinki. The participants were 679 Italian adults $(M=346 ; F=333)$, aged between 17 and 83 years $(M=33.35 ; \mathrm{SD}=12.80)$. They were recruited through convenience sampling, filled an online survey, after having expressed their consent to the participation and were free to abandon the participation at any time. They came from different Italian regions. The most part of them was single (427, 62.9\%); the remaining part was married $(177,26.1 \%)$, or in other conditions (cohabiting $=45,6.6 \%$; divorced $=26$, $3.8 \%$; widow $=4,0.6 \%)$. Almost half of them $(348,51.3 \%)$ had university degree; about the remaining half $(310,45.7 \%)$ had high school degree; 21 respondents (3\%) had junior high school degree. The $44.6 \%$ (303) were students, the $32.7 \%$ were employees $(119,17.5 \%$ in private sector; $103,15.2 \%$ in public sector); the remaining part was freelance $(66,9.7 \%)$, unemployed $(70,10.3 \%)$, or in other working conditions $(18$, $2.7 \%)$. The respondents were primarily involved in remote working activities $(144,21.2 \%)$ or smart studying activities (299, 44\%); a short percentage of them had a stop in their working activity due to the government's provisions for the coronavirus quarantine (temporarily do not work $=48,7.1 \%$; layoffs $=48,7.1 \%)$. The most part of them lived with their origin family $(409,60.2 \%)$ or with their partner $(163,24 \%)$; the remaining part lived with their family $(53,7.8 \%)$, alone $(38,5.6 \%)$ or with colleagues or fellow students $(16,2.4 \%)$. Half of them $(367,54.1 \%)$ regularly practiced physical activity before the COVID-19 lockdown; the remaining part (312, $45.9 \%$ ) did not. 


\section{Measures and procedure}

The data were collected through an anonymous online survey, collecting information on sociodemographic data, contact history with COVID-19, emotional symptoms and physical activity routines. The survey was created ad hoc for the purposes of the study. Sociodemographic data were collected on gender, age, instruction, current location, and employment status. Subsequently, in the first part, the survey regarded the assessment of individual's behavior about the adherence to social isolation and its effects. In the second part, the attitudes toward distressing situations in general were assessed. In the third part, the survey regarded the valuation of positive and negative emotions regarding the present situation, and the degree of experienced symptoms. Finally, information on habits regarding physical activity were collected. Due to the purpose of the current study, having at least 18 years old was the only inclusion criterion. Participants completed the questionnaires through an online survey platform (Google Forms). It took approximately $10 \mathrm{~min}$ to complete. To prevent double compilation, the respondents were asked to provide an individual code composed of the first three letters of the last name and the first three letters of the first name that, matched with other demographic information, permitted to check any eventual duplicate.

\section{Data analysis}

Data were performed using the SPSS v. 25. Descriptive analysis, contingency tables with Pearson's Chi-square, and medians were computed.

\section{Results}

The first part of the survey regarded the assessment of individual's behavior about the adherence to social isolation and its effects. The respondents expressed their agreement/disagreement to some statements on five-point Likert scale $(1=$ totally disagree; $5=$ totally agree). Table 1 presents the medians of the answers to the questions comprised in this section.

The second part of the survey, then, was focused on the attitudes toward distressing situations in general. The respondents indicated their responses similarly than in the previous section, ranking their answers on five-point Likert scale. Table 2 reports the medians of the answers to the items included in the second section. The third part of the survey assessed the positive and negative emotions regarding the present situation, and the degree of experienced symptoms, using a 4-point Likert scale, from $1=$ not at all to $4=$ extremely (see Table 3 ).

Analyzing the habits regarding physical activity, we have compared (Chi-square, $p<0.05$ ) those who have maintained the same habits with those who have changed their habits during the lockdown. As we can see in Table 4, the most part of the respondents have maintained their training habits $(313,46.01 \%)$; among the respondents that have changed habits, are more represented those who have started training activities $(154,32.9 \%)$ than those who have interrupted them.

Then, we have compared this result with the emotions and the symptoms experienced, exploring if the physical
Table 1 Medians of the responses to the first section

Table 2 Medians of the responses to the second section

\begin{tabular}{ll}
\hline Items & $\begin{array}{l}\text { Mdn } \\
(N=679)\end{array}$ \\
\hline I think my behavior is useful for avoiding the spread of COVID-19 & 5 \\
I really want to avoid crowds of people & 5 \\
The social contact I have with people can cause harm & 4 \\
I struggle to avoid social relationships outside the home & 2 \\
\hline
\end{tabular}

\begin{tabular}{ll}
\hline Items & $\begin{array}{l}\text { Mdn } \\
(N=679)\end{array}$ \\
\hline In the face of difficult situations, I think everything will be resolved & 4 \\
I am always positive about my future & 4 \\
I am able to keep my emotions under control & 4 \\
To check my anxieties, I can turn to a good friend or a member of my family & 4 \\
To control my anxieties, I can count on my spiritual beliefs & 3 \\
Every day I feel like I'm "in a cage" and I don't see "way out" & 2 \\
My past experiences have prepared me well to face the present moment & 3 \\
I always reach my goals & 4
\end{tabular}


Table 3 Medians of the responses to the third section

\begin{tabular}{ll}
\hline Positive emotions & $\begin{array}{l}\text { Mdn } \\
(N=679)\end{array}$ \\
\hline Energy & 3 \\
Happiness & 2 \\
Calm & 3 \\
Negative emotions & \\
Depression & 2 \\
Nervousness & 3 \\
Fear & 2 \\
Anxiety & 2 \\
Loneliness & 2 \\
Anger & 2 \\
Experienced symptoms & \\
Insomnia & 2 \\
Tachycardia & 1 \\
Disgust & 1 \\
Headache & 2 \\
Fatigue & 2 \\
Muscle tension & 2 \\
\hline
\end{tabular}

Table 4 Contingency table and Chi-square $(p<.05)$

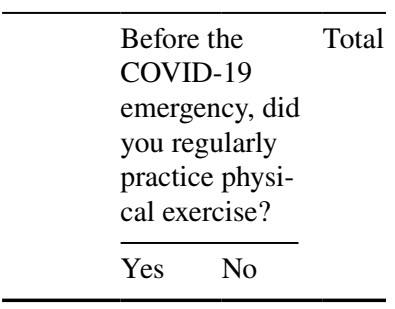

During the emergency COVID-19, did you carry out physical activity at home?

$\begin{array}{clll}\text { Yes } & 313 & 154 & 467 \\ \text { No } & 54 & 158 & 212 \\ \text { Total } & 367 & 312 & 679\end{array}$

Chi-square $=101.364, \quad$ d.f. $=1$, $p<.001$

exercise would have provided benefits to the individuals during the social isolation. We have distinguished two levels of emotions and symptoms: low level comprises answers 0 and 1 to the 4-point Likert scale; high level comprises answers 3 and 4 to the 4-point Likert scale. Surprisingly, only few significant comparisons have been found: the respondents who have maintained their training habits tend to experience - among the positive emotionssignificantly higher levels of energy and calm; among the symptoms, they tend to experience lower levels of fatigue as reported in Table 5.

Then, comparing the emotions and the symptoms in the respondents who have changed their habits during the
Table 5 Contingency table and Chi-square $(p<.05)$

\begin{tabular}{|c|c|c|c|}
\hline & \multicolumn{2}{|c|}{ Maintenance of the same habits } & \multirow[t]{2}{*}{ Tota } \\
\hline & Yes*Yes & $\mathrm{No}^{*} \mathrm{No}$ & \\
\hline \multicolumn{4}{|c|}{ Energy $^{1}$} \\
\hline Low & 71 & 59 & 130 \\
\hline High & 242 & 99 & 341 \\
\hline \multicolumn{4}{|l|}{$\mathrm{Calm}^{2}$} \\
\hline Low & 118 & 81 & 199 \\
\hline High & 195 & 77 & 272 \\
\hline \multicolumn{4}{|c|}{ Fatigue $^{3}$} \\
\hline Low & 204 & 76 & 280 \\
\hline High & 109 & 82 & 191 \\
\hline Total & 313 & 158 & 471 \\
\hline
\end{tabular}

Yes*Yes: I practiced physical activity and I have continued during the lockdown; No*No: I did not practice physical activity and I have not practiced it during the lockdown

${ }^{1}$ Chi-square $=11.29$, d.f. $=1, p<.001$

${ }^{2}$ Chi-square $=7.92$, d.f. $=1, p=.005$

${ }^{3}$ Chi-square $=12.70$, d.f. $=1, p<.001$

Table 6 Contingency table and Chi-square $(p<.05)$

\begin{tabular}{llll}
\hline & \multicolumn{2}{l}{ Changing of the habits } & Total \\
\cline { 2 - 3 } & Yes*No & No*Yes & \\
\hline Happiness ${ }^{1}$ & 44 & 92 & 136 \\
Low & 10 & 62 & 72 \\
High & & & \\
Nervousness & & & \\
Low & 17 & 73 & 118 \\
High & 37 & 154 & 208 \\
Total & 54 & &
\end{tabular}

Yes*No: I practiced physical activity, but I have not practiced it during the lockdown; No*Yes: I did not practice physical activity, but I have practiced it during the lockdown

${ }^{1}$ Chi-square $=8.35$, d.f. $=1, p=.004$

${ }^{2}$ Chi-square $=4.13$, d.f. $=1, p=.04$

lockdown (see Table 6), we have not found many significant differences between the two groups. More specifically, among the two subgroups that have changed their physical activity habits, those who have interrupted the exercise of physical activity have shown significantly higher levels of nervousness; on the other hand, those who have started training activity during the lockdown have shown higher levels of happiness.

The last analysis, reported in Table 7, compared two different subgroups: (1) those who have changed habits in exercising physical activity during the lockdown and (2) those who have maintained previous habits in physical activity during the lockdown. The findings have shown that 
Table 7 Contingency table and Chi-square $(p<.05)$

\begin{tabular}{llll}
\hline & $\begin{array}{l}\text { Changing of the } \\
\text { habits }\end{array}$ & $\begin{array}{l}\text { Maintaining the } \\
\text { habits }\end{array}$ & Total \\
\hline $\begin{array}{llll}\text { Energy } \\
\text { Low }\end{array}$ & 84 & 130 & 214 \\
High & 124 & 341 & 465 \\
Fear $^{2}$ & & 292 & 401 \\
Low & 109 & 179 & 278 \\
High & 99 & 284 & 385 \\
Anxiety & & 187 & 294 \\
Low & 101 & & 480 \\
High & 107 & 348 & 199 \\
Headache & & 123 & 382 \\
Low & 132 & & 297 \\
High & 76 & 280 & 679 \\
Fatigue & & 191 & \\
Low & 102 & 471 & \\
High & 106 & & \\
Total & 208 & &
\end{tabular}

${ }^{1}$ Chi-square $=10.93$, d.f. $=1, p=.001$

${ }^{2}$ Chi-square $=5.49$, d.f. $=1, p=.02$

${ }^{3}$ Chi-square $=8.10$, d.f. $=1, p=.004$

${ }^{4}$ Chi-square $=7.57$, d.f. $=1, p=.006$

${ }^{5}$ Chi-square $=6.35$, d.f. $=1, p=.012$

the respondents who have maintained the same habits have higher levels of positive emotions (energy), lower levels of negative emotions (fear and anxiety) and lower level of experienced symptoms (headache and fatigue).

\section{Discussion}

This study sought to offer a looking of the Italian condition in the first weeks of the restrictive stages defined by the severe diffusion of COVID-19 epidemic. The aim of this study was to survey the general community in Italy to better recognize their levels of psychological impact, emotional responses and maintaining their daily exercise or physical activity routines during the initial stage of the outbreak. Our findings suggest that the most part of the respondents have maintained their training habits; among the respondents that have changed habits, are more represented those who have started training activities than those who have interrupted them.

Exploring if the physical exercise would have provided benefits to the individuals during the social isolation, we found that the respondents who have maintained their training habits tend to experience-among the positive emotions-significantly higher levels of energy and calm; among the symptoms, they tend to experience lower levels of fatigue.

Our results highlighted that the individuals who have interrupted the exercise of physical activity have shown significantly higher levels of nervousness; on the other hand, those who have started training activity during the lockdown have shown higher levels of happiness. These findings agree with the results of previous studies that have studied the benefits of physical activity; it has a major role in mental health and cognitive function $[12,13]$, because exercise has positive effects in preventing and alleviating depression and anxiety [14, 15].

Staying physically active is a significant part of a healthy lifestyle that can avoid, or manage chronic diseases, easily perform activities of daily living (e.g., getting in and out of a chair or bed), and reserve physical function for older adults.

During major infectious disease outbreaks, lockdown was a necessary preventive measure, but it is often associated with a negative psychological effect $[6,16]$. The last findings of this study have shown that the respondents who have maintained the same habits have higher levels of positive emotions (energy), lower levels of negative emotions (fear and anxiety) and lower level of experienced symptoms (headache and fatigue). Therefore, maintaining the same habits during the lockdown could be considered as a protective factor regard the physical and psychological wellbeing. Moreover, performing home-based exercises provide an opportunity for people to maintain fitness and healthy levels, contributing significantly to total energy expenditure $[17,18]$.

\section{Conclusions}

This study has some limitations. First, our design did not consent creating a causal relationship, and prospective researches are needed to make causal inferences, using, i.e. longitudinal studies. Moreover, the use of psychometric validated scales - in place of ad hoc questions - to measure emotions or wellbeing, existent in literature, could give more opportunities to data analysis. Second, the opportunity of selection bias due to the online survey should be regarded. Third, regarding the variable "age", the absence of data analysis does not allowed you to conclude that the results were not influenced by age.

Despite some limitations, this study provides a snapshot on the initial responses during the lockdown in Italy. The COVID-19 pandemic caused significant challenges to people, families, communities and countries, but is also shown us several lessons. As the epidemic continues to spread, our findings can be an important guidance for the development of a psychological support strategy. First, by confirming the 
fact that maintaining regular physical activity and routinely exercising is an essential approach for healthy living.

Consequently, it is important to recognize the psychological influence of the pandemic to better prepare researchers to look and assess the psychosocial repercussions on the general population. Based on the results of the study, suggestions on apposite interventions can be provided to the policy makers regarding the strategies to adopt to maintain people wellbeing and to prevent health and psychological dysfunctions $[19,20]$.

Author contributions Conceptualization, DC, PM, BM and NMM; data curation, BM, MG, MC and SL; formal analysis, PM, BM, and SL; methodology, PM, and MG; supervision, DC, PM, BM, and NMM; visualization, $\mathrm{MG}$ and $\mathrm{MC}$; writing — original draft, DC, PM and BM; writing-review and editing, DC, NMM and PM. All authors have read and agreed to the published version of the manuscript.

Funding This research received no external funding.

\section{Compliance with ethical standards}

Conflicts of interest The authors declare no conflict of interest.

Ethical approval This study was carried out in accordance with the recommendations of the Ethical Code of the University of Palermo and of the Code of Ethics approved by the General Assembly of the Italian Association of Psychology held on March 27, 2015.

Human and animal rights The study is in agreement with the Declaration of Helsinki.

Informed consent All participants were informed about the trials of the study and the anonymity of their answers before providing their written consent to participate, in accordance with the Declaration of Helsinki.

\section{References}

1. Li Q, Guan X, Wu P, Wang X et al (2020) Early transmission dynamics in Wuhan, China, of novel coronavirus-infected pneumonia. N Engl J Med 382:1199-1207. https://doi.org/10.1056/ NEJMoa2001316

2. Hellewell J, Abbott S, Gimma A et al (2020) Feasibility of controlling COVID-19 outbreaks by isolation of cases and contacts. Lancet Glob Health 8:488-496. https://doi.org/10.1016/S2214 $-109 \mathrm{X}(20) 30074-7$

3. World Health Organization (2020) Responding to community spread of COVID-19: interim guidance. https://www.who.int/ docs/defaultsource/coronaviruse/20200307-responding-to-covid -19-communitytransmissionfinal.pdf. Accessed 7 Mar 2020

4. Blakey SM, Reuman L, Jacoby RJ, Abramowitz JS (2015) Tracing "Fearbola": psychological predictors of anxious responding to the threat of ebola. J Cogn Psychother 39(6):816-825. https:// doi.org/10.1007/s10608-015-9701-9

5. Leung GM (2003) The impact of community psychological responses on outbreak control for severe acute respiratory syndrome in Hong Kong. J Epidemiol Community Health 57:857863. https://doi.org/10.1136/jech.57.11.857
6. Brooks SK, Webster RK, Smith LE et al (2020) The psychological impact of quarantine and how to reduce it: rapid review of the evidence. Lancet 395:912-920. https://doi.org/10.1016/S0140 $-6736(20) 30460-8$

7. Huang Y, Zhao N (2020) Generalized anxiety disorder, depressive symptoms and sleep quality during COVID-19 epidemic in China: a web-based cross-sectional survey. MedRxiv. https://doi. org/10.21203/rs.3.rs-17172/v1

8. Wang C, Pan R, Wan X, Tan Y, Xu L, Ho CS, Ho RC (2020) Immediate psychological responses and associated factors during the initial stage of the 2019 coronavirus disease (COVID-19) epidemic among the general population in china. Int J Environ Res Public Health 17(5):1729. https://doi.org/10.3390/ijerph17051729

9. Jiménez-Pavón D, Carbonell-Baeza A, Lavie CJ (2020) Physical exercise as therapy to fight against the mental and physical consequences of COVID-19 quarantine: special focus in older people. Prog Cardiovasc Dis. https://doi.org/10.1016/j.pcad.2020.03.009

10. Pedersen BK, Saltin B (2015) Exercise as medicine-evidence for prescribing exercise as therapy in 26 different chronic diseases. Scand J Med Sci Sports 25(S3):1-72. https://doi.org/10.1111/ sms. 12581

11. Powell KE, Paluch AE, Blair SN (2011) Physical activity for health: what kind? How much? How intense? On top of what? Annu Rev Public Health 32(1):349-365. https://doi.org/10.1146/ annurev-publhealth-031210-101151

12. Guicciardi M, Crisafulli A, Doneddu A, Fadda D, Lecis R (2019) Effects of metabolic syndrome on cognitive performance of adults during exercise. Front Psychol 10:1845. https://doi.org/10.3389/ fpsyg.2019.01845

13. Bangsbo J, Blackwell J, Boraxbekk CJ et al (2019) Copenhagen consensus statement 2019: physical activity and ageing. Br J Sports Med 53:856. https://doi.org/10.1136/bjsports-2018-100451

14. Schuch FB, Vancampfort D, Richards J, Rosenbaum S, Ward PB, Stubbs B (2016) Exercise as a treatment for depression: a metaanalysis adjusting for publication bias. J Pshyciatrc Res 77:42-51. https://doi.org/10.1016/j.jpsychires.2016.02.023

15. Di Corrado D (2017) Biological underpinnings of mood and the role of physical exercise. J Sport Health Sci 13(3):461-468. https ://doi.org/10.1007/s11332-017-0374-5

16. Sood S (2020) Psychological effects of the coronavirus disease-2019 pandemic. RHiME 7:23-26

17. Hammami A, Harrabi B, Mohr M, Krustrup P (2020) Physical activity and coronavirus disease 2019 (COVID-19): specific recommendations for home-based physical training. Manag Sport Leis. https://doi.org/10.1080/23750472.2020.1757494

18. Francavilla G, Francavilla C (2013) Physical exercise is terapy. Med Sport 66:625-628

19. Gasparro R, Scandurra C, Maldonato NM, Dolce P, Bochicchio V, Valletta A, Sammartino G, Sammartino P, Mariniello M, Espedito di Lauro A, Marenzi G (2020) Fear of COVID-19 and depression among Italian dentists: the mediating role of perceived job insecurity. Int J Environ Res Public Health 17:5338. https://doi. org/10.3390/ijerph17155338

20. Maldonato NM, Bottone M, Chiodi A, Continisio GI, De Falco R, Duval M, Muzii B, Siani G, Valerio P, Vitelli R, Scandurra C (2020) A mental health first aid service in an Italian University Public Hospital during the Coronavirus Disease 2019 outbreak. Sustainability 12:4244. https://doi.org/10.3390/su12104244

Publisher's Note Springer Nature remains neutral with regard to jurisdictional claims in published maps and institutional affiliations. 